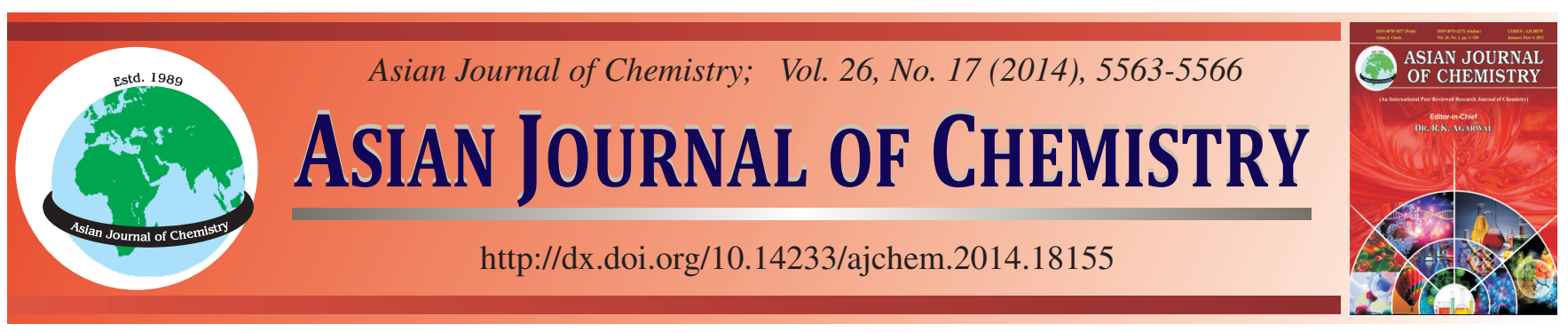

\title{
Electromagnetic Wave Absorbing Properties of Double-Layer Cement Composite and Theory Analysis $\dagger$
}

\author{
Yuefang Zhang ${ }^{1,2}$, Wanjun HaO ${ }^{2, *}$, BaOyi Li $^{3}$, Yuping Duan ${ }^{1}$ and Shunhua LiU ${ }^{1}$
}

${ }^{1}$ School ofMaterials and Engineering, Dalian University of Technology, Dalian 116085, P.R. China

${ }^{2}$ School of Materials Science and Chemical Engineering, Hainan University, Haikou 570228, P.R. China

${ }^{3}$ The 33st Institute of China Electronics Technology Group Corporation, Taiyuan 030002, P.R. China

*Corresponding author: E-mail: hwj8899@163.com

\begin{abstract}
In this paper, we have developed double-layer cement matrix composites by using impedance matching design. Expanded polystyrene beads were used as an electromagnetically transparent material in the composites. The composites' electromagnetic wave absorption properties (filled with carbon black, manganese dioxide and $\mathrm{Ni}-\mathrm{Zn}$ ferrite at $2.6-18 \mathrm{GHz}$ ) were examined. The results showed that the expanded polystyrene beads significantly improved the impedance matching characteristics between the composites and the free space and the scattering properties of expanded polystyrene beads contributed to further wave attenuation. We further optimized the cement matrix's absorption properties by altering the ratios of fillers, absorbent types and specimen thicknesses. A maximum absorption of -22.3 $\mathrm{dB}$ at $4.8 \mathrm{GHz}$ (bandwidth $11.4 \mathrm{GHz},<-10 \mathrm{~dB}$ ) was achieved under the following conditions: matching layer of 60 vol $\%$ expanded polystyrene and $4 \mathrm{vol} \%$ ferrite, absorbing layer of $50 \mathrm{vol} \%$ expanded polystyrene and $6 \mathrm{vol} \%$ carbon black and a specimen thickness of $20 \mathrm{~mm}$.
\end{abstract}

Keywords: Cement matrix composites, Absorption properties and components, Electromagnetic transparent materials, Double-layer.

\section{INTRODUCTION}

The use of wireless telecommunication systems and highfrequency circuit devices has increased dramatically over the years. Electronic devices emit electromagnetic waves that cause serious electromagnetic interference (EMI) and wave pollution. Therefore, absorbers of electromagnetic waves are greatly needed.

Cement-based materials, which are readily available with good environmental adaptability, are the most commonly used structural material in construction as mentioned by Cao and Chung ${ }^{1}$. Cement is minimally conductive, but its wave absorption capacities are also low. A practical method to increase the absorption effectiveness of cement composites is by introducing fillings or loadings as shown by Guan et al. ${ }^{2}$. Xiong et $a l .{ }^{3}$ and Pourjavadi et al. ${ }^{4}$ introduced nanometric $\mathrm{TiO}_{2}$ and nanoSiO $\mathrm{S}_{2}$ particles, respectively, as fillings to improve the absorbing performance of cement-based materials. Yamane et al..$^{5}$ developed a wide-band, ferrite electromagnetic wave absorber panel for building walls. With these fillings, cement composites can achieve reflection losses as great as $-8 \sim-20$
$\mathrm{dB}$ in specific frequencies. However, the narrow absorption band, high density and forbidding cost limit their applications. Moreover, the mismatching impedance between the surfaces of cement composites and surrounding free space hinders the improvement of wave absorption properties. Therefore, Guan et al. ${ }^{6}$ proposed a new method that involves the filling of electromagnetically transparent beads, such as expanded polystyrene, into cement. Kimura and Hashimoto ${ }^{7}$ investigated a three-layer wave absorber using common building materials for wireless LAN. Oikonomou et al. ${ }^{8}$ studied a hexagonal, ferrite, multilayer microwave absorber for impedance matching. Chen et al. ${ }^{9}$ investigated the influence of $\mathrm{SiO}_{2}$ fillers on microwave absorption properties of carbonyl iron/carbon black doublelayer coatings. Little progress, however, has been made on multi-layer cement-based composites.

In this paper, we have developed a double-layer cement matrix composite composed of a matching layer and an absorbing layer. Expanded polystyrene exhibits low density, high specific strength, low water absorption and it served as a poor electromagnetic filler in this study. The absorption components tested herein include carbon black (CB), manganese dioxide 
$\left(\mathrm{MnO}_{2}\right)$ and $\mathrm{Ni}-\mathrm{Zn}$ ferrite (Ni-Zn), each with different attenuation mechanisms as demonstrated by Guan et al. ${ }^{10}$ and Li et al. ${ }^{11}$. These three absorbers were used in combination to increase the overall absorption capacities of the cement composite. Furthermore, the attenuation mechanisms for double-layer absorbers are discussed and the main influence factors are given.

\section{EXPERIMENTAL}

Portland cement of Type P.O42.5R is used in this study. The expanded polystyrene beads have a loose density of 0.02 $\mathrm{g} / \mathrm{cm}^{3}$ and an average diameter of $2 \mathrm{~cm} . \mathrm{MnO}_{2}$ and $\mathrm{Ni}-\mathrm{Zn}$ ferrite $\left(\mathrm{Ni}_{0.5} \mathrm{Zn}_{0.5} \mathrm{Fe}_{2} \mathrm{O}_{4}\right)$ purchased from Shenzhen Bertrand Metal Co. were analytically pure. The $\mathrm{N} 234$ carbon black was milled in a ball milling for $3 \mathrm{~h}$ prior to use.

To prepare the absorbing layer, the cement was mixed with water in a mortar mixer at a ratio of 0.35 (water/cement). The absorbent powder and the expanded polystyrene beads were added into the paste and then mixed until uniformly distributed in the material. Then, the paste was poured into the oiled moulds sized $200 \mathrm{~mm} \times 200 \mathrm{~mm}$ and then smoothed with a float. After the initial setting of the bottom layer, the matching layer of equal thickness was added. The specimen was demolded one day later and cured at room temperature for 28 days. All contents used in this study are volumetric ratios.

The electromagnetic parameters of the cement were tested by the coaxial flange method using an Agilent $8720 \mathrm{~B}$ vector network analyzer. The electromagnetic absorption properties of double-layer wave absorbing material were tested in an anechoic chamber using the arched testing method over 2.6$18 \mathrm{GHz}^{12}$.

\section{RESULTS AND DISCUSSION}

Electromagnetic properties of cement: Fig. 1 (a) and (b) show the frequency dependence of complex permeability $\left(\mu=\mu^{\prime}-\mathrm{j} \mu^{\prime \prime}\right)$ and permittivity $\left(\varepsilon=\varepsilon^{\prime}-\varepsilon^{\prime \prime}\right)$ for raw and cured cement, respectively. It can be seen that the $\mu$ has changed after 28 days of curing, while $\mu^{\prime}$ and $\mu^{\prime \prime}$ are close to 1 and 0 , respectively. In contrast, the $\varepsilon^{\prime}$ is maintained at about 5 and slightly decreases, while the $\varepsilon^{\prime \prime}$ increases from 0.1 to 0.2 . This phenomenon is probably due to the generation of a low silicate level through complex reactions between water and cement during the 28 days of curing. This suggests that the cements' absorption capacity increases upon dielectric loss resulting from the polarization of metal oxides and minerals within the material. However, this type of cured cement still has a poor absorption capacity. Therefore, additional absorbers with a high $\varepsilon^{\prime \prime}$ or $\mu$ " are needed to provide new wave attenuation mechanisms in this composite. Furthermore, cement has a uniform and relatively compact structure that inhibits incidental wave transmission and therefore, in order to suppress the reflection from the cement composites surface, additions with the capability of adjusting the impedance matching characteristics are also needed.

Theoretical analysis of double-layer absorber: A good absorber is defined by two important criteria as discussed by Lin et al. ${ }^{13}$. First, "matched characteristics impedance" should be obtained by making the intrinsic impedance of the absorber equal to the impedance of the free space. This requires that
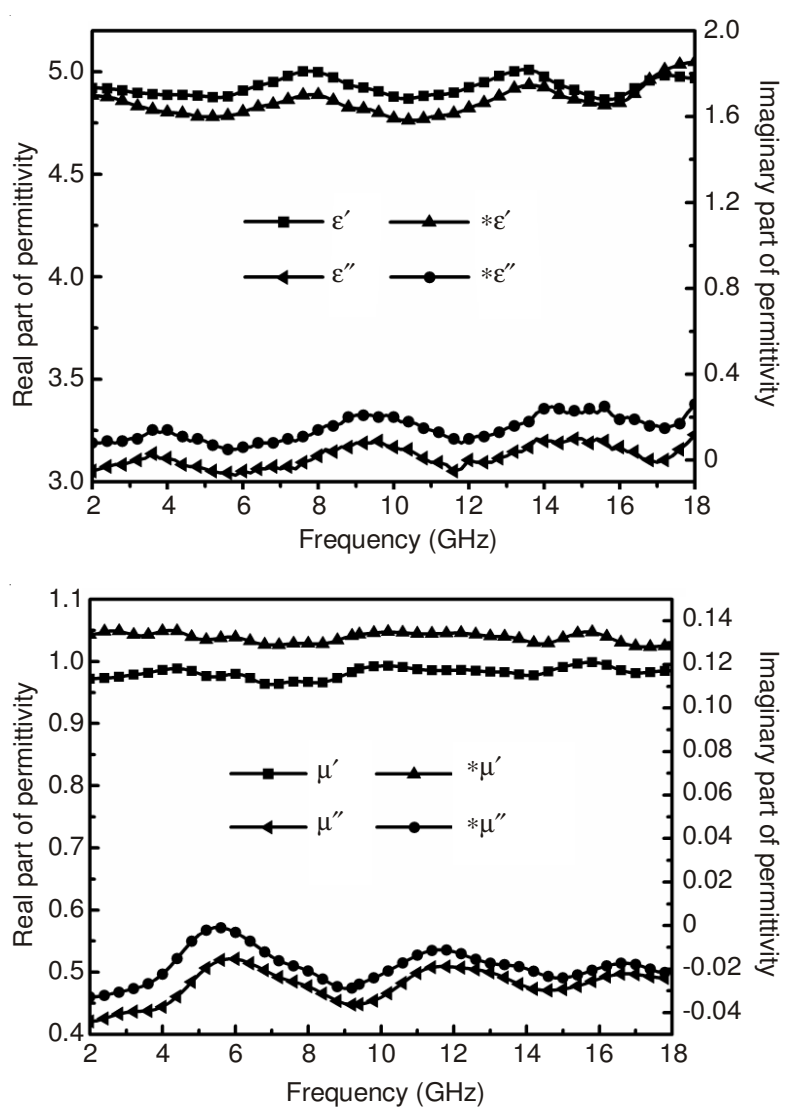

Fig. 1. Complex permittivity (a) and permeability (b) of cement and cement after curing for 28 days (noted by $*$ )

the $\mu$ and $\varepsilon$ should be as close as possible. Second, the incident wave should be rapidly attenuated through the absorber. Thus, these two criteria are incompatible in a uniform absorber. Therefore, a double-layer absorber design is needed in order to achieve good impedance matching and good absorption capacity simultaneously.

For a double-layer absorber backed by a perfect conductor, the input impedance $Z_{\text {in }}$ of the absorber is given by eqn. $1^{14}$ :

$$
\mathrm{Z}_{\text {in }}=\mathrm{Z}_{1} \frac{\mathrm{Z}_{1} \tan \mathrm{h}\left(\mathrm{k}_{1} \mathrm{~d}_{1}\right)+\mathrm{Z}_{2} \tanh \left(\mathrm{k}_{2} \mathrm{~d}_{2}\right)}{\mathrm{Z}_{1}+\mathrm{Z}_{2} \tanh \mathrm{h}\left(\mathrm{k}_{1} \mathrm{~d}_{1}\right) \tan \mathrm{h}\left(\mathrm{k}_{2} \mathrm{~d}_{2}\right)}
$$

where $Z_{i}=\sqrt{\frac{\mu_{0} \mu_{i}}{\varepsilon_{0} \varepsilon_{i}}}, k_{i}=\omega \sqrt{\varepsilon_{i} \varepsilon_{0} \mu_{i} \mu_{0}}, Z_{i} k_{i}$ and $d_{i}$ are the characteristic impedance, the propagation constant and the thickness of the ith layer, respectively. According to the transmission line theory, the reflection loss (RL) is expressed as follows ${ }^{15}$ :

$$
R L=20 \log \left|\frac{Z_{\text {in }}-Z_{0}}{Z_{\text {in }}+Z_{0}}\right|(d B)
$$

where $Z_{0}$ is the characteristic impedance of a vacuum, described as $Z_{0}=\sqrt{\frac{\mu_{0}}{\varepsilon_{0}}}=120 \pi \Omega$. As seen from eqns. 1 and 2, the absorption properties of the double-layer absorber can be modulated by multiple parameters, including $\mathrm{m}\left(\mu=\mu^{\prime}-\right.$ $\left.\mathrm{j} \mu^{\prime \prime}\right), \varepsilon\left(\varepsilon=\varepsilon^{\prime}-\varepsilon^{\prime \prime}\right)$ and $\mathrm{d}$ of the matching layer and the absorbing layer to make $\mathrm{Z}_{\text {in }}$ closer to $\mathrm{Z}_{0}$. 
TABLE-1

SAMPLE DESIGN (\%vol.)

\begin{tabular}{ccccccc}
\hline Sample & 1 & 2 & 3 & 4 & 5 & 6 \\
\hline Matching layer & $60 \% \mathrm{EPS}$ & $60 \% \mathrm{EPS}$ & $60 \% \mathrm{EPS}+4 \% \mathrm{CB}$ & $60 \% \mathrm{EPS}+4 \% \mathrm{MnO}_{2}$ & $60 \% \mathrm{EPS}+4 \%$ Ferrite & $60 \% \mathrm{EPS}$ \\
Absorbing layer & $50 \% \mathrm{EPS}+2 \% \mathrm{CB}$ & $50 \% \mathrm{EPS}+6 \% \mathrm{CB}$ & $50 \% \mathrm{EPS}+6 \% \mathrm{CB}$ & $50 \% \mathrm{EPS}+6 \% \mathrm{CB}$ & $50 \% \mathrm{EPS}+6 \% \mathrm{CB}$ & $6 \% \mathrm{CB}$ \\
\hline
\end{tabular}

Influence of matching layer on absorption properties: Table-1 shows the samples' \#1-\#6 matching layer and absorbing layer designs. Fig. 2 shows the reflection loss of samples \#2 \#5, where all samples have the same thickness of $20 \mathrm{~mm}$. The samples' absorbing layers are uniformly filled with $50 \%$ expanded polystyrene and $6 \%$ carbon black and the matching layers are filled with $60 \%$ expanded polystyrene (\#2), $60 \%$ expanded polystyrene and $4 \%$ carbon black (\#3), $60 \%$ expanded polystyrene and $4 \% \mathrm{MnO}_{2}(\# 4), 60 \%$ expanded poly-styrene and $4 \% \mathrm{Ni}-\mathrm{Zn}$ Ferrite (\#5), respectively. From the cures, the reflection loss of all our samples are significantly improved over previous reports ${ }^{3,4,7}$. Most noticeably, sample \#5 has a peak value of $-22.3 \mathrm{~dB}$ at $4.8 \mathrm{GHz}$ with an absorption bandwidth (below $-10 \mathrm{~dB}$ ) of $11.4 \mathrm{GHz}$.

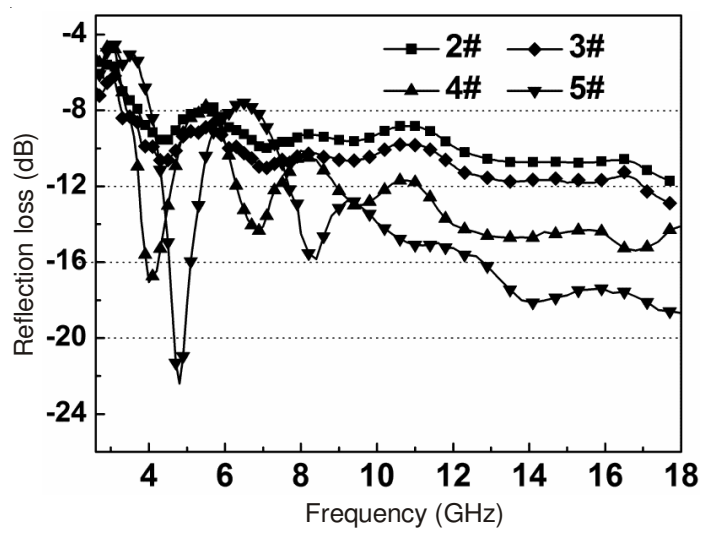

Fig. 2. Reflection losses of samples with the same absorbing layers and different matching layers

The improvement of absorption properties is primarily attributable to the filling of expanded polystyrene. According to the effective medium theory, expanded polystyrene decreases the of the composite, which means there is less reflection on the absorber's surface. The incident wave is reflected instead and scattered by the porous structure that is composed of expanded polystyrene beads and cement coated walls, whose attenuation effectiveness is described as follows ${ }^{16}$ :

$$
\mathrm{I}(\mathrm{x})=\mathrm{I}_{0} \exp \left(-\mathrm{n} \frac{\mathrm{k}^{4}|\chi|^{2}}{6 \pi} \mathrm{x}\right)
$$

where $\mathrm{k}^{4}|\chi|^{2} / 6 \pi=\sigma_{\mathrm{SC}}$ is the scattering cross section, is the beads number in one unit volume and is the polarizability. In this case, the attenuation effectiveness is determined by the permittivity, permeability and the polarizability of the composite. The expanded polystyrene filling also leads to phase shift as waves transmitting from one bead to another and thus contributes to electromagnetic attenuation. Secondly, the additional absorbents in the matching layers also enhance electromagnetic attenuation. As we can see from eqn. 3, the absorbent filling enhances attenuation through scattering. However, the input impedance is also changed and it greatly affects the reflection loss. Carbon black is considered as a conductive powder and $\mathrm{MnO}_{2}$ is a promising dielectric loss absorbent, both of which have large $\varepsilon$ and small $\mu$. According to eqn. 1, a large $\varepsilon$ and a small $\mu$ may deteriorate the impedance matching characteristic of the composite and hinder the decrease of reflection loss. In contrast, Ni-Zn ferrite has been widely used in absorbing materials due to its large e and. We consistently observed an increase in the composite attenuation ability by filling Ni-Zn Ferrite. The reflection of incident waves is also suppressed by similar values of $\varepsilon$ and $\mu$. Thus, the reflection loss is greatly improved (Fig. 2). It is concluded that Ni-Zn ferrite and other ferrous, magnetic materials are suitable as additional absorbents in matching layers of cement composites.

Influence of absorbing layer on absorption properties: Fig. 3 shows the reflection loss of samples \#1, \#2 and \#6 with the same matching layers and $20 \mathrm{~mm}$ thickness. The absorbing layers are filled with $50 \%$ expanded polystyrene and $2 \%$ carbon black (\#1), $50 \%$ expanded polystyrene and $6 \%$ carbon black (\#2) and $6 \%$ carbon black (\#6), respectively. The reflection loss decreases as the frequency increases from 2.6-18 GHz. The reflection loss of sample \#2 is below $-8 \mathrm{~dB}$ in 3.8-5.4 and 5.8-18 GHz, which is much better than the other two.

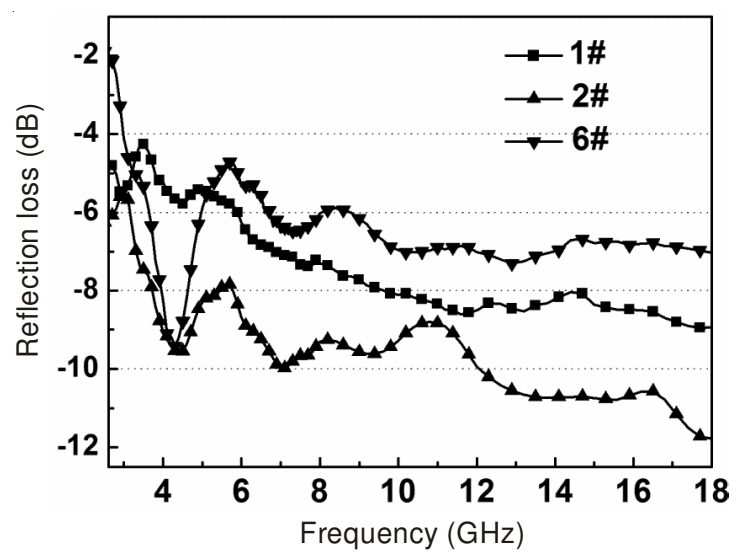

Fig. 3. Reflection losses of samples with the same matching layers and different absorbing layers

The incident wave would be reflected partly on the interface of matching layer and absorbing layer. So, the matched characteristics impedance is also required for the absorbing layer. Otherwise, the absorption properties will deteriorate from the strong reflection, such as sample \#6 which doesn't include expanded polystyrene in the absorbing layer. At the same time, strong absorption ability is especially needed in the absorbing layer, which is mainly determined by the carbon black particle content. When the carbon black concentration is low, a contactless distribution of carbon black limits the attenuation effectiveness by resistance losses. An increase in the carbon black concentration forms a conductive network with the narrowing space among neighboring particles, through which the incident wave will be severely attenuated. However, a further increase 
in carbon black concentration negatively impacts the absorption properties. This is probably due to the agglomeration phenomenon of the carbon black and the mismatching of impedance with the matching layer. The optimal concentration of carbon black is predicted to be beyond $6 \%$, as shown in Fig. 3. Therefore, sample \#2 obtains excellent absorption properties (below $-10 \mathrm{~dB}$ ) with a bandwidth of $6 \mathrm{GHz}$.

Influence of thickness on absorption properties: Fig. 4 shows a typical relationship between the reflection loss and frequency for sample \#3 with different thicknesses. The reflection loss is improved as the thickness increases and their peak values [-9.7 dB at $12.3 \mathrm{GHz}(10 \mathrm{~mm}),-10.8 \mathrm{~dB}$ at $11.6 \mathrm{GHz}$ $(15 \mathrm{~mm})$ and $-10.6 \mathrm{~dB}$ at $9.6 \mathrm{GHz}(20 \mathrm{~mm})$, respectively] are obtained. The increase in thickness is effectively an increase in the content of expanded polystyrene beads and absorbents, so the reflection loss can be improved as long as the impedance matches with the free space.

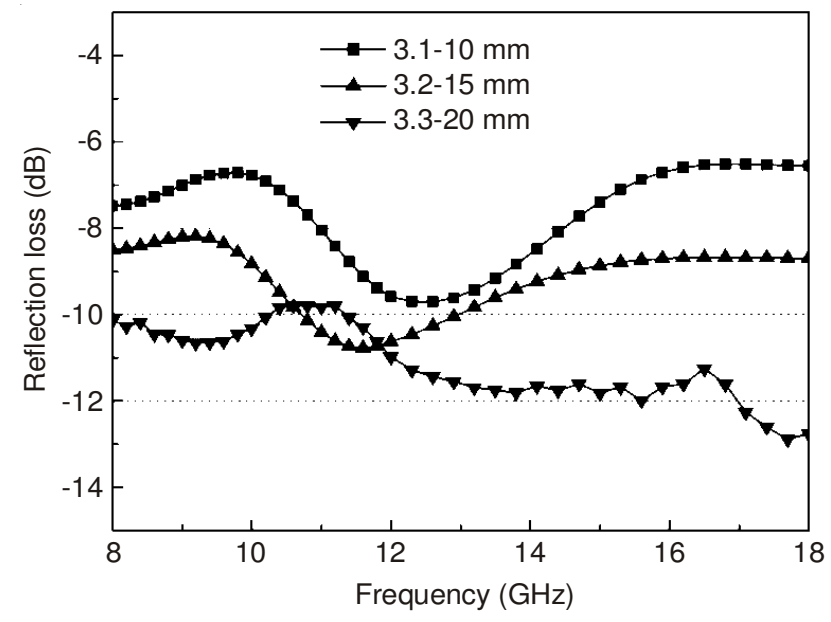

Fig. 4. Reflection losses of samples 3 with different thicknesses

\section{Conclusion}

By designing matching layers and absorbing layers, we prepared a double-layer cement matrix composite with excellent absorption properties. We found that expanded polystyrene beads can not only adjust the impedance matching characteristic of composites but can also attenuate the incident wave by scattering. Ni-Zn ferrite is suitable as an additional absorbent in the matching layer and a carbon black concen- tration of $6 \%$ can decrease the reflection loss at their best in the absorbing layer. The increase in thickness can also improve absorption properties within a certain range. When the matching layer is filled with $60 \%$ expanded polystyrene and $4 \%$ ferrite and the absorbing layer is filled with $50 \%$ expanded polystyrene and $6 \%$ carbon black, the sample with a thickness of $20 \mathrm{~mm}$ can have the highest peak value of $-22.3 \mathrm{~dB}$ at $4.8 \mathrm{GHz}$, with an absorption bandwidth (below $-10 \mathrm{~dB}$ ) of $11.4 \mathrm{GHz}$.

\section{ACKNOWLEDGEMENTS}

The authors acknowledged the support from the National Science and Technology support plan for twelfth five years of China [No: 2012BAJ02B08-3], the Natural Science Foundation of Hainan province [No. 510205], the Major projects of haiku city [No. 2012004].

\section{REFERENCES}

1. J. Cao and D.D.L. Chung, Carbon, 41, 2433 (2003).

2. H. Guan, S. Liu, Y. Duan and J. Cheng, Cement Concr. Compos., 28, 468 (2006).

3. G. Xiong, L. Xu and M. Deng, J. Funct. Mater. Devices, 11, 87 (2005) (in Chinese).

4. A. Pourjavadi, S.M. Fakoorpoor, A. Khaloo and P. Hosseini, Mater. Design., 42, 94 (2012).

5. T. Yamane, S. Numata and T. Mizumoto, Int. Symp. Electromag. Compatibility, 2, 799 (2002).

6. H. Guan, S. Liu, Y. Duan and Y. Zhao, Cement Concr. Compos., 29, 49 (2007).

7. K. Kimura and O. Hashimoto, Electron. Lett., 40, 1323 (2004).

8. A. Oikonomou, T. Giannakopoulou and G. Litsardakis, J. Magn. Magn. Mater, 316, 827 (2007).

9. L. Chen, Y. Duan, L. Liu, J. Guo and S. Liu, Mater. Des., 32, 570 (2011).

10. H. Guan, Sh. Liu, Y. Zhao and Y. Duan, J. Electron. Mater, 35, 892 (2006).

11. L. Baoyi, D. Yuping, Z. Yuefang and L. Shunhua, Mater. Des., 32, 3017 (2011).

12. B. Li and S. Liu, Research on the Absorbing Property of Cement Matrix Composite Materials, International Conference on Concrete Construction, Kingston University, London. Excellence in Concrete Construction Through Innovation, pp. 215-219 (2009).

13. H. Lin, H. Zhu, H. Guo and L. Yu, Mater. Res. Bull., 43, 2697 (2008).

14. E. Michielssen, J.-M. Sajer, S. Ranjithan and R. Mittra, Microw. Theory Technol., 41, 1024 (1993).

15. M.R. Meshram, N.K. Agrawal, B. Sinha and P.S. Misra, J. Magn. Magn. Mater, 271, 207 (2004).

16. F.D. Ge, J. Zhu and L.M. Chen, Tien Tzи HsuehPao, 24, 82 (1996). 